\title{
Fenofibric acid: a new fibrate approved for use in combination with statin for the treatment of mixed dyslipidemia
}

This article was published in the following Dove Press journal:

Vascular Health and Risk Management

12 May 2010

Number of times this article has been viewed

\author{
Peter Alagona Jr \\ Penn State Heart and Vascular \\ Institute, Penn State College of \\ Medicine, Hershey, PA, USA
}

Correspondence: Peter Alagona Jr Program Director General Cardiology, Penn State Heart and Vascular Institute, Associate Professor of Medicine and Radiology, Penn State College of Medicine, PO Box 850, MC H047, 500 University Drive, Room C5833, Hershey, PA I7033, USA

$\mathrm{Tel}+\mathrm{I} 717-53 \mathrm{I}-1790$

Fax + I 7|7-53|-|793

Email palagona@hmc.psu.edu

\begin{abstract}
The last two to three decades have seen an explosive growth in interest and information regarding cardiovascular disease (CVD) risk assessment and treatment. Evidence for the role of low-density lipoprotein (LDL) in risk has led to a series of clinical guidelines/ recommendations on the importance of LDL lowering with statin treatment. There is also substantial evidence on a number of lipoproteins in the initiation and progression of atherosclerosis and CV events. Health care professionals have not embraced easily novel approaches to identifying those at increased risk and more aggressive treatment. This is especially true for non-LDL factors. The use of non-statin drugs such as fibrates has been modest and many health care professionals avoid consideration of combination therapy due to an inordinate fear of toxicity. This review will attempt to provide appropriate background information on lipids/ lipoproteins, including non-high density lipoprotein and risk, as well as data available on fibrates and combination pharmacologic therapy. We will review a new agent, TriLipix ${ }^{\circledR}$ (fenofibric acid), and its potential role in treatment.
\end{abstract}

Keywords: dyslipidemia, peroxisome proliferated activated receptors, fibrate, fenofibric acid, TriLipix, very-low-density lipoprotein, triglycerides, non-high density lipoprotein

\section{Introduction}

Traditional laboratory evaluation used in clinical medicine includes measurement of total cholesterol (TC), triglycerides (TG), low-density lipoprotein (LDL) and high-density lipoprotein (HDL). Cholesterol is the most abundant steroid in animal tissue. The other major neutral lipid is formed by combining a three carbon glycerol (an alcohol) with three fatty acids (acyl groups) to form triacylglycerol, also known as TG. TG are the major form of circulating fat and are used as a prime source of energy. These hydrophobic lipids (Figure 1) are poorly soluble and have to be transported in the aqueous plasma by a variety of lipoproteins. The core of plasma lipoproteins contain cholesterol ester (CE) and TG surrounded by phospholipids with their more soluble polar phosphate groups pointing out to the surface of the particle. Lipoproteins also contain one or more apoproteins on their surface which are major determinates of the biochemical and metabolic activities of these particles (Figure 2). Dietary TG are absorbed via the intestine and transported in chylomicrons. Endogenous production also occurs in the liver. TG enter the circulation from the liver mainly in the core of very-low-density lipoprotein (VLDL).

Laboratory assessment and subsequent clinical use of plasma lipid measurements have provided an important means for CVD risk assessment and a guide to treatment. Standard laboratory tests measure neutral lipids; however, CE and TG, contained submit your manuscript | www.dovepress.com

Dovepress

6714 


\section{STEROL}
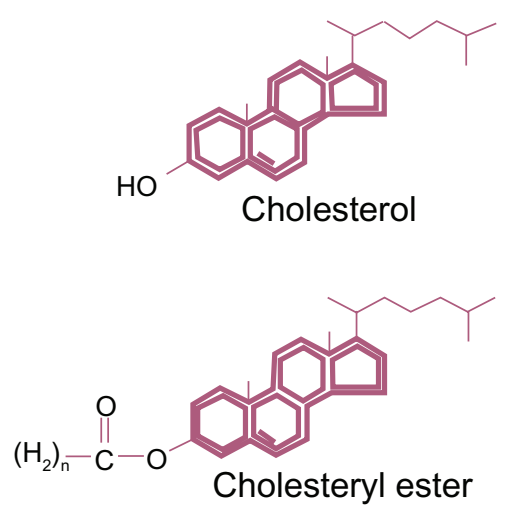

TRIGLYCERIDE

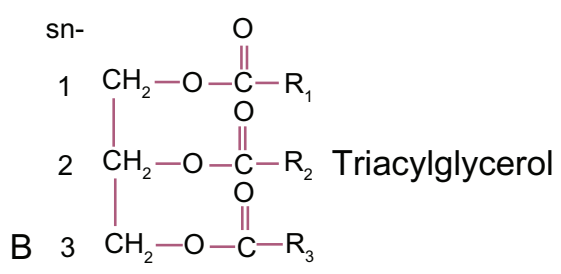

Figure I The neutral or non-polar plasma lipids.

within various circulating lipoproteins, do not provide information on the number, biochemical or metabolic characteristics of specific particles. We have been trained to relate CVD risk to TC and LDL; however the Framingham study revealed the mean LDL of subjects who developed CV events versus those who did not during long term follow-up was not significantly different. ${ }^{1}$ The National Cholesterol Education Program (NCEP) Adult Treatment Panel (ATP) has made three major consensus statements which include recommendations for lipid goals in patients at risk ${ }^{2}$ (Table 1). However we do not define what constitutes a "normal" LDL in specific individuals. Definition of "elevated" LDL and determination to treat with LDL-lowering strategies are based on risk stratification using the presence of other concomitant traditional risk factors. We do not recommend LDL lowering in adults without grossly elevated LDL unless they also have other risk factors present. ${ }^{3}$ The Framingham Risk Score assigns points for sex, age, cigarette smoking, systolic blood pressure, TC, and HDL. The total number of points for any individual is used to estimate their "absolute risk" of developing a $\mathrm{CV}$ event over the ensuing 10 years. High risk is defined as $>20 \%$, intermediate $10 \%$ to $20 \%$ and low risk $<10 \%$. The NCEP ATP modified and simplified the Framingham approach but again based treatment recommendations on the number of risk factors present. The initial NCEP ATP III recommendations included an LDL goal of $<100 \mathrm{mg} / \mathrm{dL}$ in those with prior CV events. However based on the results of aggressive LDL-lowering trials utilizing higher doses and more potent statins providing further RRR ${ }^{4-6}$ the ATP III panel in 2002 produced a "footnote" with the statement that in the "highest risk group of patients an LDL goal of $\leq 70 \mathrm{mg} / \mathrm{dL}$ is optional". ${ }^{7}$ However the same primary author had earlier raised concern over what appeared to be attenuation of more aggressive LDL lowering with statins on relative risk reduction (RRR) when previous recommended LDL goals had already been attained. ${ }^{8}$ The continued occurrence of the majority of predicted $\mathrm{CV}$ events, even in those aggressively treated and attaining LDL goals, is now termed residual risk. This issue was again raised in a controversial analysis by Hayward et al published in 2006 raising a question of whether more aggressive LDL lowering with higher dosage and more potent statin treatment was supported by the data. The authors evaluated published statin trial data and calculated the number-needed-to-treat (NNT) to prevent a $\mathrm{CV}$ event in the secondary risk population when reducing LDL from $>200 \mathrm{mg} / \mathrm{dL}$ to $100 \mathrm{mg} / \mathrm{dL}$ to be $1: 20$. However, the NNT obtained by further reducing LDL from $100 \mathrm{mg} / \mathrm{dL}$ to $70 \mathrm{mg} / \mathrm{dL}$ or less was $1: 67$, comparable to the RRR noted in primary prevention trials using statins. ${ }^{9,10}$ Their conclusion was that the data was not robust enough to warrant the more aggressive optional treatment recommendation of $\mathrm{LDL} \leq 70 \mathrm{mg} / \mathrm{dL}$ due to potential for increased side effects, toxicity and cost. ${ }^{11}$

Multiple lipoproteins, all of which transport CE and TG, depending on their surface apoproteins, may have variable atherogenic, protective, or both, potentials. Penetration and retention of apoB particles, including LDL, in the subendothelial space appear to initiate a process leading to inflammation, plaque formation and eventually atherothrombotic events. ${ }^{12}$ However, other lipoproteins and inflammatory factors obviously contribute to the process and more information regarding specific lipoproteins and their behavior may further improve risk stratification and treatment. The atherogenic dyslipidemia characterized by increased TG and decreased HDL is now commonly associated with increased waist circumference, metabolic syndrome (MS) and type II diabetes. ${ }^{13} \mathrm{CV}$ events and mortality are increased in these dysmetabolic states and may be related to non-LDL factors.

\section{Triglycerides}

A number of hereditary/familial disorders featuring or including increased TG play a role in CVD risk. Familial hypertriglyceridemia (FHTG) is a fairly common inher- 


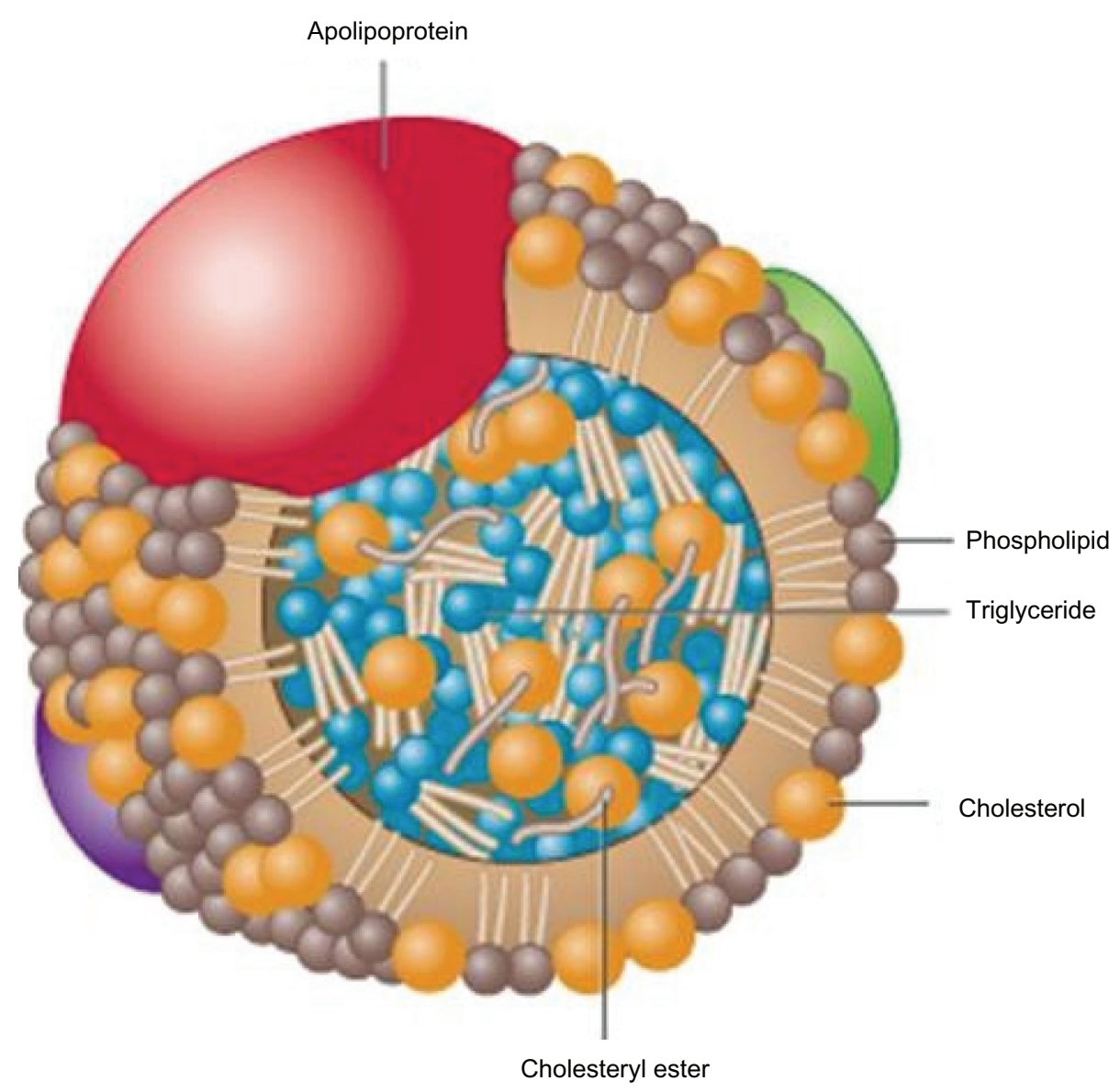

Figure 2 Apo B lipoprotein with neutral lipids contained in the core.

Reproduced with permission from Koschinsky ML, Marcovina SM. In: Ballantyne, CM, editor. Clinical Lipidology: A Companion to Braunwald's Heart Disease. Philadelphia: Saunders Elsevier; 2009:130-143.13 Copyright (C) 2009 Elsevier.

ited disorder of uncertain origin. TG are commonly 200 to $1000 \mathrm{mg} / \mathrm{dL}$ with normal or mildLy elevated cholesterol ( $>240 \mathrm{mg} / \mathrm{dL}$ ). LDL is not usually increased. It appears to be inherited as an autosomal dominant trait but is not usually expressed until adulthood. It occurs in $1 / 500$ people. Familial dysbetalipoproteinemia or type III hyperlipoproteinemia creates a defect in apoE leading to accumulation of abnormal elevated chylomicrons and VLDL in the plasma. Patients have both increased TG and TC, usually to a similar degree. HDL is usually within normal limits. These patients can have tuboeroeruptive xanthomas (elbows, knees or buttocks) and

Table I Optimal lipid goals in high risk patients.

\begin{tabular}{lll}
\hline & Male & Female \\
\hline LDL & $<100 \mathrm{mg} / \mathrm{dL}$ & $<100 \mathrm{mg} / \mathrm{dL}$ \\
HDL & $>40$ & $>50$ \\
TG & $<150$ & $<150$ \\
Non-HDL & $<130$ & $<130$ \\
\hline
\end{tabular}

Notes: Consistent with published recommendations from the NCEP ATPIII, American Heart Association and American Diabetes Association.

Abbreviations: HDL, high-density lipoprotein; LDL, low-density lipoprotein; TG, triglycerides. palmar xanthomas. Premature coronary artery disease (CAD) is common and aggressive evaluation and treatment, including combination therapy, is often needed. Familial combined hyperlipidemia (FCHL) is a mixed dyslipidemia with moderately elevated fasting TG, TC and decreased HDL. Hepatic overproduction of VLDL is the common basis of this familial defect which may occur in $1 / 200$ persons, commonly in those with premature CAD. Fasting TG in the range of 200 to $800 \mathrm{mg} / \mathrm{dL}$ and TC 200 to $400 \mathrm{mg} / \mathrm{dL}$ are common. Prominent family history supports the diagnosis. Significantly elevated apoB levels, disproportionate to LDL, is often noted. Patients with FCHL require aggressive treatment and follow-up due to significant CVD and event risk.

Increased fatty acid levels occur with increased waist circumference, obesity, MS, diabetes and post-prandial lipemia which stimulate hepatic production and secretion of VLDL resulting in increased serum TG. This in turn has a significant effect on the number, size, composition and density of almost all lipoproteins with increased number of VLDL remnant particles, including intermediate density lipoprotein (IDL) and small dense LDL particles. This 
results from the inability of lipoprotein lipase to adequately delipidate (exchange and remove CE and TG from the core of lipoproteins) VLDL and properly clear the remnant particles from the circulation. HDL is similarly affected with the production of increased small, denser particles with less Apo A-I, increased catabolic rates and therefore reduced HDL. This process may also lead to increased numbers of, with a variety of isoform heterogeneity, lipoprotein(a) [Lp(a)] particles. Lp(a) has a complex structure consisting of an LDL particle covalently bound to a glycoprotein, apolipoprotein(a) [apo(a)]. The apo(a) contains multiple repeated kringle sequences similar to the sequence found in the fibrinolytic proenzyme plasminogen. This similarity of the LDL-Apo(a) or $\mathrm{Lp}(\mathrm{a})$ to plasminogen might provide a link between the processes of atherosclerosis (LDL) and thrombosis. The plasminogen-like apo(a) competes with plasminogen and inhibits it's fibrinolytic activity. However the varying $\mathrm{Lp}(\mathrm{a})$ isoforms, difficulty with accurate and reproducible laboratory measurement, resistance of $\mathrm{Lp}$ (a) lowering with pharmacologic treatment and the lack of outcome data continue to make approaches to Lp(a) speculative at best. ${ }^{13}$ Even excluding the possible role of $\mathrm{Lp}(\mathrm{a})$, from the clinical standpoint the overall result of this dyslipidemia is enhancement of the atherogenic potential of the circulation. ${ }^{14}$

Although TG appear to play a role in CV events, severely elevated TG $(>500 \mathrm{mg} / \mathrm{dL})$ are associated with increased risk of acute pancreatitis and at very high levels may increase risk of in situ thrombosis.

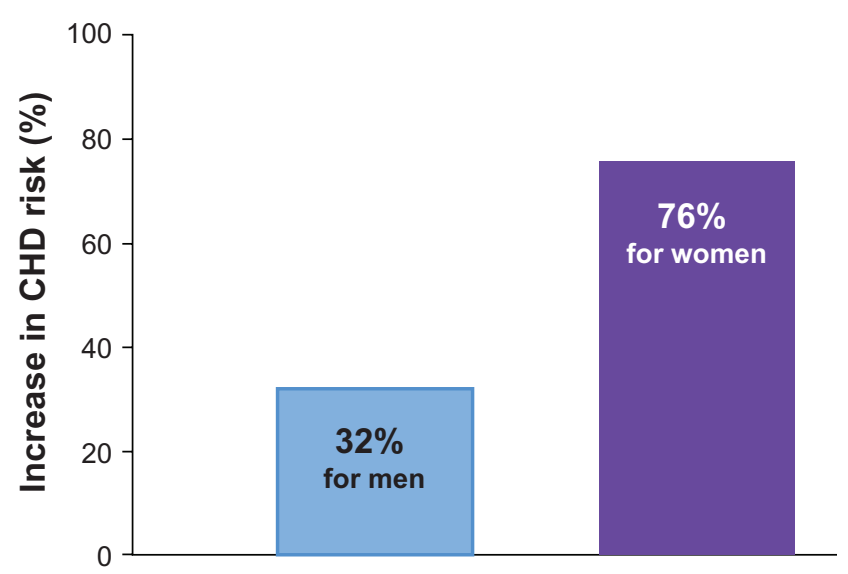

Figure 3 Increases in triglycerides associated with increases in CHD risk: a metaanalysis of 17 prospective studies.

Notes: For every increase in serum TG level of $89 \mathrm{mg} / \mathrm{dL}$, the risk of CHD increases $32 \%$ in men and $76 \%$ in women. The 17 prospective studies reporting association between elevated TG and CV endpoints included 46,4I3 men with 2445 events (average follow-up 8.4 years) and 10,864 women with $439 \mathrm{CV}$ events (average follow-up II.4 years).

Abbreviations: CHD, coronary heart disease; CV, cardiovascular; TG, triglycerides.
Although beyond the scope of this review, lifestyle modification to address a variety of dysmetabolic states, including certain dyslipidemias and risk, associated with elevated TG is always part of the therapeutic strategy. Diet, exercise and decreased alcohol intake when necessary can quickly and dramatically lower free fatty acids and $\mathrm{TG}$, resulting in decreased non-HDL and VLDL.

\section{Non-LDL risk}

The Framingham Heart Study has provided cogent data consistent with the important role of HDL in CVD risk. A $1 \mathrm{mg} /$ $\mathrm{dL}$ difference in HDL level is inversely associated with a $1 \%$ to $2 \%$ difference in CV risk. ${ }^{15-17}$ The important role of elevated TG in CVD risk is supported by the often quoted large meta-analysis by Hokanson and Austin ${ }^{18}$ (Figure 3 ) in addition to data from the 10-year study of the Caerphilly and Speedwell Cohorts. ${ }^{19}$ The Copenhagen Male Study ${ }^{20}$ followed 2906 men without known CVD for 8 years and The Prospective Cardiovascular Munster Study (PROCAM) Study ${ }^{21} 4849$ men for up to 8 years, all without known CVD. These studies consistently reveal the relationship between TG and CV events independent of other known major risk factors as well as HDL. These data, however, are just as consistently questioned because the tightest relationship occurs when also associated with either elevated LDL or decreased HDL. Genest described the prevalence of a variety of lipoprotein disorders in 102 kindreds $(n=603)$ with premature coronary artery disease (CAD) documented by coronary arteriography. ${ }^{22}$ More than half of the patients had a familial lipoprotein disorder with the most common being hypertriglyceridemia with hypoalphalipoproteinemia (decreased apo A-1), excess Lp(a) and combined hyperlipidemia (Figure 4). Genest et al also reviewed the role of LDL, HDL and TG in men with premature CAD and found that both initial TG and HDL levels, but not LDL correlated with significant angiographic disease $^{23}$ and followed this with further investigation indicating the need for more aggressive evaluation of lipoprotein status in these patients. An appropriate and logical conclusion is that no single lipoprotein parameter will identify the majority of patients at risk and with significantly increased LDL, although a strong association with the development of CAD is actually an infrequent finding in patients with premature CAD.

Increased TG-rich lipoproteins have significant adverse effects on HDL composition, number, size, density, apoA-1 content and function. Recent reanalysis of CARE and PROVE-IT ${ }^{24}$ reveal that recurrent clinical events even after aggressive LDL lowering are directly related to HDL and TG levels, respectively. Non-HDL (TC-HDL) now appears to be a more powerful CVD risk predictor than $\mathrm{LDL}^{25}$ treatment 
recommendations for non-HDL lowering have been developed and included in the ATP III recommendations. Non-HDL is directly related to serum TG levels and is an indicator of the number of apoB atherogenic particles including VLDL and its remnant particles LDL, IDL, and $\mathrm{Lp}(\mathrm{a})$. It is not uncommon to have patients with LDL that does not appear significantly elevated or is at the NCEP ATPIII recommended goal but continue to have an elevated non-HDL $(30 \mathrm{mg} / \mathrm{dL}$ greater than LDL). This population requires more aggressive treatment aimed at further decreasing the number of apoB particles, which can be addressed in a number of ways.

\section{Combination pharmacologic treatment}

Clinical trials utilizing non-statin or combination therapy (statin + fibrate), especially in specific populations (MS, diabetes, elevated TG and non-HDL, decreased HDL), have revealed significant $\mathrm{CV}$ event reductions in both the primary and secondary risk groups. The Helsinki Heart Study, a primary prevention trial in hypercholesterolemic subjects, included more then 4000 men without known CVD. Over a 4-year follow-up those treated with gemfibrozil exhibited increased HDL with reductions in TG, non-HDL, and LDL. There was a $35 \%$ decrease in CV events in the treated group. Those with high LDL:HDL ratios $(>5)$ and elevated TG $(>200 \mathrm{mg} / \mathrm{dL})$ had the highest event rates and greatest benefit from treatment. ${ }^{26}$ The Bezafibrate Post-Infarction Study evaluated subjects in this highest risk group for recurrent events. In those with elevated TG and fitting criteria for MS, bezafibrate significantly reduced recurrent $\mathrm{CV}$ events versus placebo. ${ }^{27}$ The Stockholm Ischaemic Heart Disaese Study treated subjects post-mycardial infarction (MI) with clofibrate plus niacin versus placebo. Coronary heart disease death was reduced by $36 \%$ and total mortality by $26 \%$ over 5 years ${ }^{28}$ without evidence of increased muscle or hepatic toxicity. The VA-HIT Study randomized more than 2200 post-MI subjects with baseline mean LDL $111 \mathrm{mg} / \mathrm{dL}$ and HDL $32 \mathrm{mg} / \mathrm{dL}$. The treatment group received $1200 \mathrm{mg} /$ day of a long-acting gemfibrozil daily without concomitant statin. Mean TG decreased by $30 \%$ and HDL increased by $6 \%$. The treatment group had a reduction in the primary end-point, mortality and non-fatal MI by $24 \% .{ }^{29}$ Patients with diabetes exhibited the greatest reduction. The controversial FIELD Trial used fenofibrate in patients with diabetes but without known CAD or typical diabetic dyslipidemia (elevated TG, decreased HDL). Fenofibrate did not significantly reduce the risk of the primary outcome of coronary events. It did reduce total CV events $(13.9 \%$ to $12.5 \% ; P=0.035)$ due to fewer non-fatal MI and revascularization. Major reductions in laser treatment for retinopathy, occurrence of albuminuria, and amputations were documented. ${ }^{30}$ In March 2010 the Effects of Combination Lipid Therapy in Type 2 Diabetes Mellitus, an NIH study with support from multiple industry partners, was published. ${ }^{31} 5518$ patients with diabetes who were being treated with open label simvastatin were randomized to either simvastatin/fenofibrate or simvastatin/placebo. Subjects had known clinical CVD or diabetes plus 2 or more risk factors. After a mean follow-up of 4.7 years there was no difference in the primary end-point of non-fatal MI, non-fatal stroke or $\mathrm{CV}$ death between the groups. The combination of statin/ fenofibrate did not increase RRR for events. These studies do not support the routine use of combination treatment in patients with diabetes; however, the concern about both the

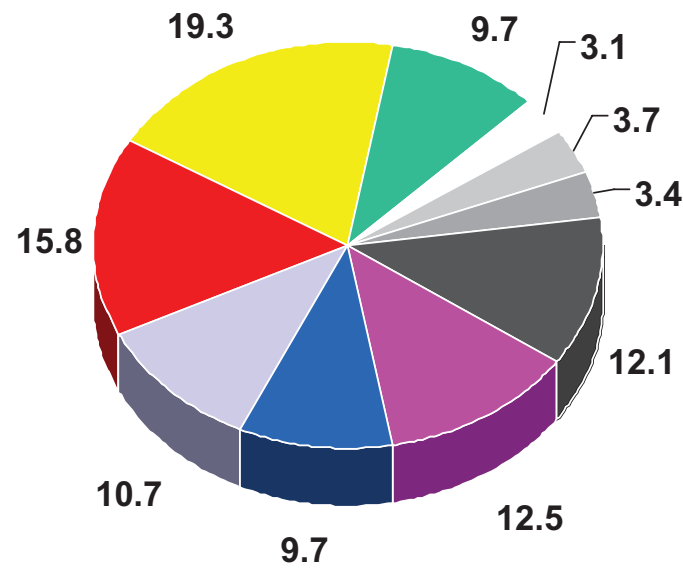

\begin{tabular}{|l|}
\hline TG+HDL 9.7 \\
$\square$ LDL+TG+HDL 3.1 \\
LDL+HDL 3 \\
LDL+TG 3.4 \\
LDL 12.1 \\
Normal 12.5 \\
High TG 9.7 \\
Hyperapobetalipoproteinemia 10.7 \\
Lp(a) Excess 15.8 \\
Low HDL 19.3
\end{tabular}

Figure 4 Prevalence of dyslipidemias in men $<60$ years with angiographic evidence of coronary atherosclerosis. Drawn from data of Genest et al. ${ }^{23}$ Notes: This figure reveals a variety of lipid/lipoprotein abnormalities in patients with premature coronary artery disease. Significantly elevated plasma LDL is not commonly seen.

Abbreviations: HDL, high-density lipoprotein; LDL, low-density lipoprotein; Lp(a), lipoprotein a; TG, triglycerides. 
FIELD and the ACCORD lipid studies is that neither evaluated patients with significant diabetic/atherogenic dyslipidemia, especially considering background prior treatment with statin. Superko and King compared several clinical end-point and angiographic regression studies for efficacy in reducing $\mathrm{CV}$ events (Figure 5). Across a variety of trials using combination lipid-modifying therapy produced an average RRR of $71.6 \%$ and a NNT of 9.6. Analysis of these data reveal significantly enhanced efficacy for event reduction with combination LDL and non-HDL lowering (decreased TG and increased HDL) compared with LDL lowering with statins alone. ${ }^{32}$ Confronted with the limited current maximized RRR of statin monotherapy for LDL lowering (Figure 6), it is time to recognize the potential of a more comprehensive and aggressive approach to global lipid/lipoprotein modification with combination therapy for CVD prevention.

\section{Fibrate therapy}

Fibrates have been used clinically since the late 1960s. This drug group includes clofibrate, bezafibrate, gemfibrozil, ciprofibrate and most recently fenofibrate. The fibrates are synthetic ligands that bind to nuclear peroxisome proliferator-activated receptors (PPAR). The PPARs are nuclear transcription factors that potentially play a role in the regulation of hundreds of genes. There are three receptor isoforms including PPAR-alpha $(\alpha)$, PPAR-gamma $(\gamma)$ and PPAR-delta $(\delta)$ encoded by different genes. They are ligand-regulated nuclear hormone receptors and when activated by an agonist result in increased gene transcription, messenger RNA production and protein synthesis. The PPARs appear to play a significant role as sensors and regulators of lipid metabolism. ${ }^{33}$ Fatty acids and eicosanoids are natural PPAR ligands. Fibrates and thiazolidinediones (TZD) are potent PPAR ligands and have well documented effects on lipid and glucose metabolism in addition to atherosclerosis and vascular inflammation. $\operatorname{PPAR}(\alpha)$ appears to play an important role in fatty acid metabolism. Mechanisms that lead to TG lowering include suppression of hepatic apo-CIII gene expression and stimulation of lipoprotein lipase (LPL) transcription, ${ }^{34}$ which promote increased cellular fatty acid uptake, oxidation and decreased production. Subsequently increased catabolism of TG-rich lipoproteins including VLDL and its remnant particles occurs, leading to decreased TG and non-HDL levels. Upregulation of apo A-I and A-II increase plasma HDL. ${ }^{35} \operatorname{PPAR}(\alpha)$ activation may improve HDL function, including reverse cholesterol transport and anti-inflammatory activity. ${ }^{36}$ Fibrates also reduce the magnitude and duration of post-prandial lipemia in those with hypertrycleridemia. Additional effects include anti-inflammatory actions with decreased in vitro expression of vascular adhesion molecule-1, and suppression of monocyte-macrophage migration ${ }^{37,38}$ and chemoattractant protein-1 (MCP-1), and are associated with reduced inflammatory markers including hsCRP, lipoprotein-associated phospholipase A2 (Lp-LPA2), interleukin-6, fibrinogen and tumor necrosis factor-alpha. ${ }^{39,40}$ Fenofibrate increased adiponectin levels, improved insulin sensitivity and significantly improved flow-mediated dilator response to hyperemia, a measure of endothelial function, in hypertriglyceridemic and MS patients. ${ }^{41,42}$ Whether all of these metabolic effects play a role in reducing atherosclerosis and $\mathrm{CV}$ risk remains in question.

Concern over potential toxicity of combination lipid-modifying therapy along with limited outcome data available has discouraged use by health care professionals even in those in the highest-risk group for recurrent CV events. Combination lipid treatment does not have documented significant increased side effects or toxicity especially in comparison to combination treatment for other $\mathrm{CV}$ conditions. Currently $30 \%$ of Americans with hypertension meet blood pressure goals $(<140 / 90)$ and the average number of drugs required in uncomplicated cases is 2.4 . This number increases to 3.4 in those with diabetes or renal dysfunction. Current guidelines recommend 3 drugs in those with systolic heart failure and diabetes patients often require multiple agents for acceptable glucose and hemoglobin $\mathrm{A}_{1 \mathrm{c}}$ control. These drug classes and combinations have been associated with frequent side effects and occasional toxicity. There are few data, and in fact no powerful retrospective or prospective studies or randomized trials that reveal significant increased risk for hepatic, renal or muscle toxicity with any lipid-modifying combination therapy. There is a small increase in muscle toxicity when using statin/gemfibrozil combination. This risk has not been documented with statins and other fibrates. ${ }^{43-45}$ There is little published information on safety and toxicity of nicotinic acid or high dose omega-3 use with fibrates. However, clinical experience and reports in small patient groups have not revealed questionable signals or increased toxicity. Fenofibrate also appears to be effective and safe when used with ezetimibe alone ${ }^{46}$ or with the combination pharmaceutical ezetimibe/simvastatin. ${ }^{47}$

Mild or modest increases in serum creatinine levels have been documented with fenofibrate and ciprofibrate. ${ }^{48,49}$ These increases were reversible during the course of the trials and the mechanism appears to be increased 
creatinine production and not a decrease in glomerular filtration rate (GFR). ${ }^{50,51}$ Subjects with GFR of 30 to $60 \mathrm{~mL} /$ $\mathrm{min} / 1.73 \mathrm{~m}^{2}$ in the Veterans Affairs High-density Lipoprotein Intervention Trial (VA-HIT) exhibited no change in GFR with gemfibrozil treatment compared to placebo after 5 years, including those with diabetes or MS. There were transient increases in creatinine in $10 \%$ of the subjects in the treatment group and $4 \%$ in the placebo group which were not statistically significant. ${ }^{52}$ Fibrates do not appear to increase the risk of acute renal dysfunction and have been shown to reduce progression of microalbuminuria in those with type II diabetes. ${ }^{49,53}$ The National Kidney Foundation and the National Lipid Association recommend that in stage 2 or greater chronic kidney disease fibrate monotherapy should be used with caution and the dose of fenofibrate reduced for those with GFR 30 to $60 \mathrm{~mL} / \mathrm{min} / 1.73 \mathrm{~m}^{2}$, and avoided in end-stage renal disease. Gemfibrozil may have some modest benefit in those with GFR less than 30 $\mathrm{mL} / \mathrm{min} / 1.73 \mathrm{~m}^{2}$; however fenofibrate appears safer when used in combination with statins. Davidson et al provide a comprehensive evidenced-based presentation of safety issues related to fibrate therapy and review and summarize all the issues. ${ }^{45}$

Fenofibric acid is not an inhibitor of the CYP P450 and has no significant drug-drug interactions in this regard. Gemfibrozil and fenofibrate when used with coumarin-type anticoagulants may prolong the prothrombin time and international normalized ratio (INR). Routine INR determinations and follow-up, as always, are recommended with dose adjustment as necessary. However, it does not appear that fibrates in general represent a unique or significant problem for this interaction.

Fibrates most pronounced effects on serum lipids include a $30 \%$ to $50 \%$ reduction in fasting TG as well as reducing the duration of post-prandial lipemia by decreasing fatty acid synthesis and increasing lipoprotein lipase activity. The higher the baseline TG the greater the reductions may be ${ }^{54-56}$ As a result there is a significant decrease in

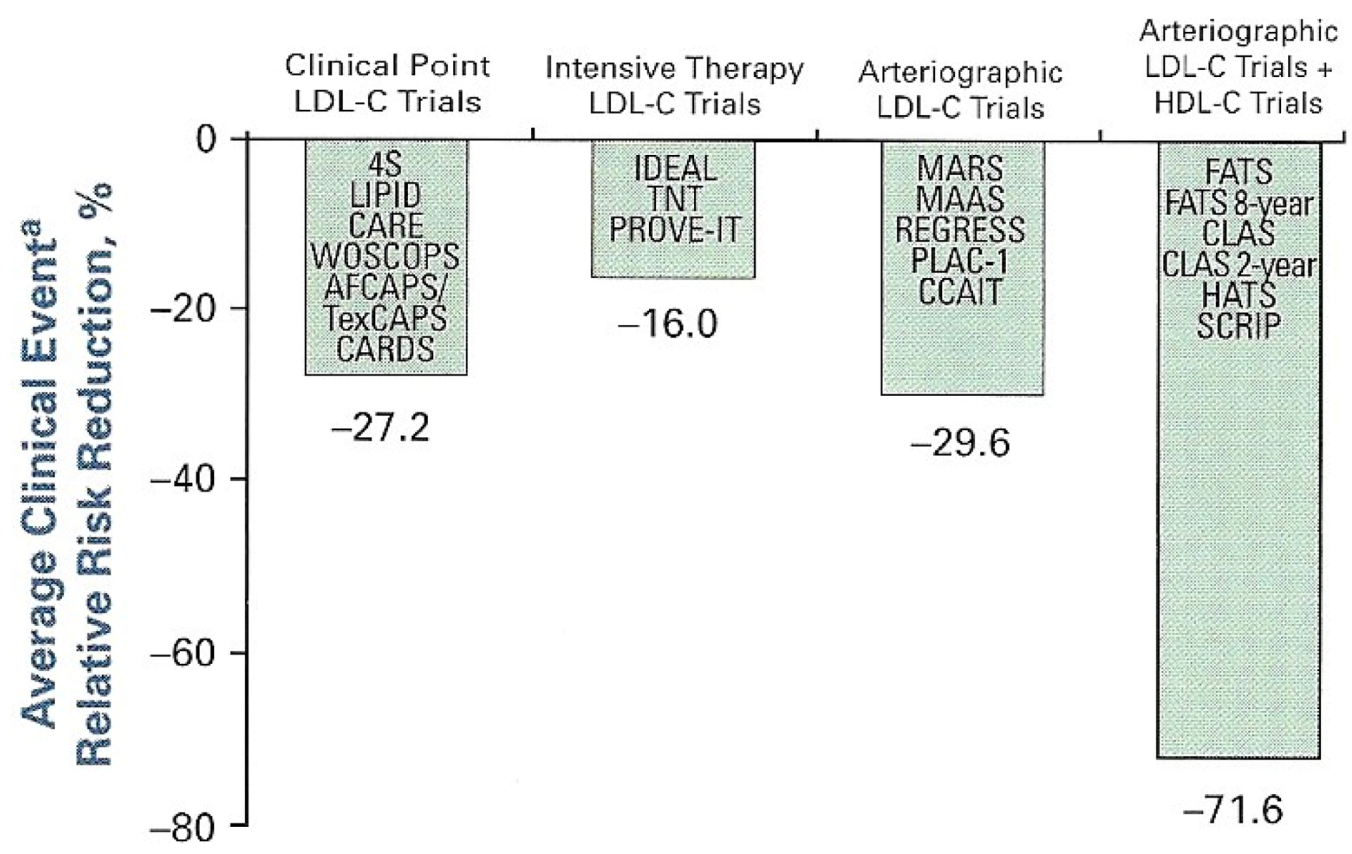

\begin{tabular}{|rrrrr|}
\hline Average & & & & \\
NNT & 41.0 & 64.6 & 64.3 & 9.6 \\
NNT/y & 8.5 & 19.1 & 28.7 & 3.4 \\
\hline
\end{tabular}

Figure 5 The average RRR for CV events in the secondary population even in the most intensive statin studies is $30 \%$. This leaves significant residual risk for recurrent events. Superko and King ${ }^{32}$ compared a variety of studies utilizing LDL lowering versus LDL lowering and HDL elevation. This latter combined approach resulted in projected RRR of $71 \%$ to $6 \%$ with a NNT of 9.6 overall and 3.4 year.

Abbreviations: CV, cardiovascular; HDL-C, high-density lipoprotein cholesterol; LDL-C, low-density lipoprotein cholesterol; Lp(a), lipoprotein a; NNT, number needed to treate; RRR, relative risk reduction; TG, triglycerides. 


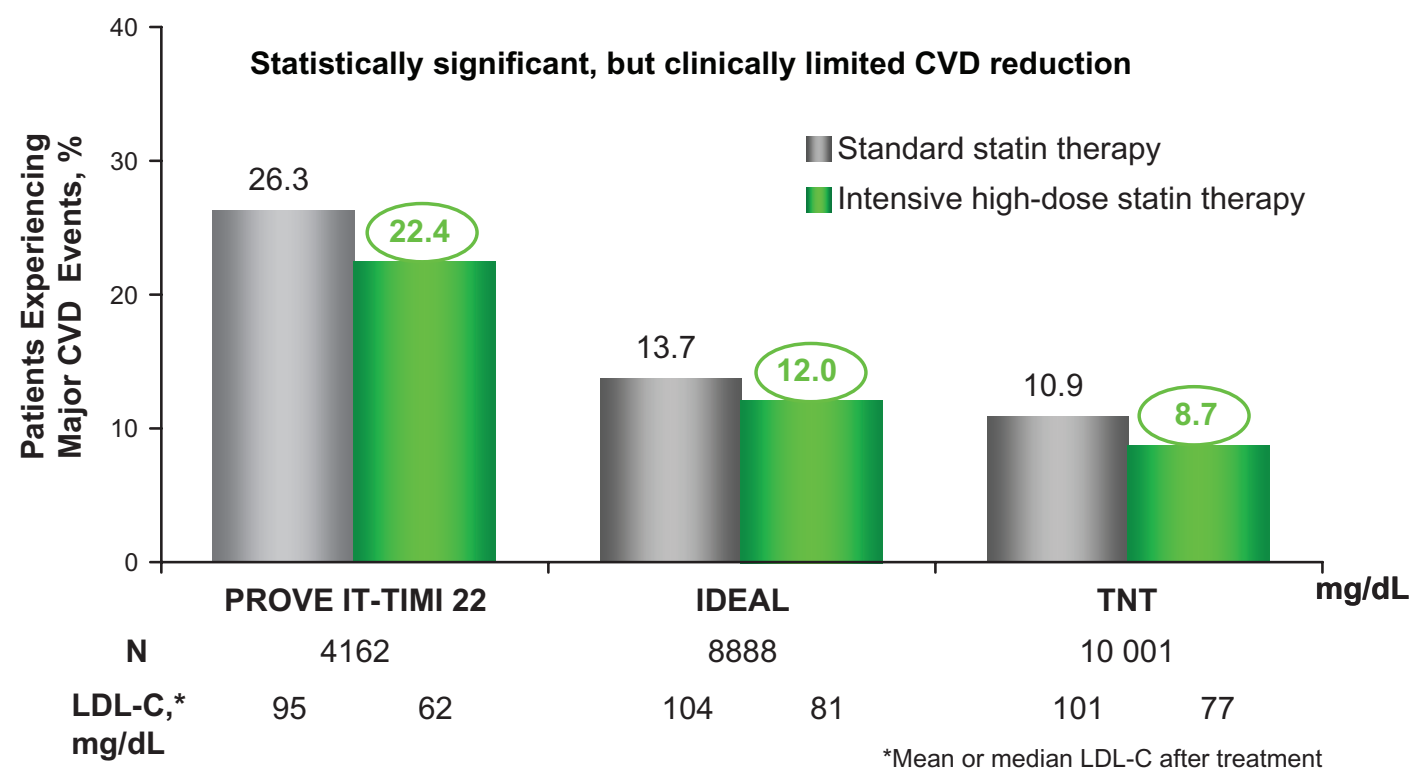

Figure 6 Residual CVD risk in patients treated with intensive statin therapy.

A closer look at 3 trials investigating intensive LDL-C lowering with statin therapy in patients with CHD revealed that residual CVD risk remains in these patients even after aggressive LDL cholesterol lowering therapy. All 3 trials compared LDL-C lowering to $\sim 100 \mathrm{mg} / \mathrm{dL}$ with more intensive LDL-C lowering to $\sim 70 \mathrm{mg} / \mathrm{dL}$ as a means of preventing major CVD events in patients with a history of CHD or acute coronary syndromes. In the Pravastatin or Atorvastatin Evaluation and Infection TherapyThrombolysis in Myocardial Infarction 22 (PROVE IT-TIMI 22) study ( $\mathrm{N}=4162$ ), pravastatin $40 \mathrm{mg}$ reduced LDL-C to $95 \mathrm{mg} / \mathrm{dL}$ and atorvastatin $80 \mathrm{mg}$ reduced LDL-C to 62 $\mathrm{mg} / \mathrm{dL}$ in patients who had been hospitalized for an acute coronary syndrome. After 2 years, $22.4 \%$ of patients treated with intensive statin therapy (atorvastatin $80 \mathrm{mg} / \mathrm{dL}$ ) suffered a major CVD event. ${ }^{4}$ In the Incremental Decrease in End Points Through Aggressive Lipid Lowering (IDEAL) study (N = 8888), simvastatin 20 mg reduced LDL-C to $104 \mathrm{mg} / \mathrm{dL}$ and atorvastatin $80 \mathrm{mg}$ reduced LDL-C to $81 \mathrm{mg} / \mathrm{dL}$ in patients with a history of acute myocardial infarction. After 4.8 years, $12.0 \%$ of patients experienced a major CVD event even after intensive LDL-C lowering with statin therapy (atorvastatin $80 \mathrm{mg}$ ). ${ }^{6}$ Finally, in the Treating to New Targets (TNT) study ( $\mathrm{N}=10,00 \mathrm{I}$ ), I0 mg atorvastatin reduced LDL-C to $101 \mathrm{mg} / \mathrm{dL}$ and $80 \mathrm{mg}$ atorvastatin reduced LDL-C to $77 \mathrm{mg} / \mathrm{dL}$ in patients with stable CHD. After 4.9 years, a major CVD event occurred in $8.7 \%$ of patients receiving intensive statin therapy $\left(80 \mathrm{mg}\right.$ atorvastatin). ${ }^{5}$

These 3 trials reveal that significant residual CVD risk remains in patients even after intensive statin treatment.

Abbreviations: CVD, cardiovascular disease; CHD, coronary heart disease; HDL-C, high-density lipoprotein cholesterol; LDL-C, low-density lipoprotein cholesterol.

the production of TG-rich lipoproteins, VLDL and its remnant particles ${ }^{57}$, and commonly an increase in HDL. Reducing TG-rich lipoproteins reduces cholesterol ester transfer protein-mediated exchange of TG and CE between lipoproteins. This reduces TG enrichment of HDL, leading to decreased metabolic modification by hepatic lipase, increased HDL particle size and decreased catabolism. Fibrates also increase hepatic apo-A1 production and HDL precursors. These mechanisms increase HDL levels anywhere from 5\% to $30 \%$ and may improve HDL function.

Fibrates have limited TC- and LDL-lowering efficacy. They may reduce LDL $10 \%$ to $20 \%$ in patients with hypercholesterolemia and normal baseline TG. ${ }^{54-57}$ A corresponding $10 \%$ to $20 \%$ reduction in apoB levels also occurs. When TG levels are elevated fibrate treatment may be associated with an increase in LDL cholesterol whether calculated by the Friedewald formula or directly measured. Although this may raise concern, because of our current LDL-centric approach to risk stratification and treatment guidelines, it is due to a decrease in TG and an increase in $\mathrm{CE}$ content in the core of LDL and other apoB particles, resulting in larger LDL particle size with a decrease in particle number. ${ }^{58,59}$ This, along with a decrease in VLDL, apoB levels and non-HDL, reduce atherogenic risk and CV events.

In recent years a number of studies have been published on the safety of the most commonly used lipid-modifying treatment, statins, with fenofibrate. Ellen and McPherson evaluated 80 patients with combined hyperlipidemia and either known CAD or with 3 or more risk factors. They received low-dose statin (pravastatin $20 \mathrm{mg}$ or simvastatin $10 \mathrm{mg}$ ) and fenofibrate (300 mg or 200 micronized form) for a total of 220.6 patient-years on combination therapy. When fenofibrate was used combined with low-dose statin a marked improvement in all lipid measurements was observed. The decrease in the TC/HDL ratio, considered a reliable indicator of CV event risk, was $24 \%$ with fenofibrate alone, $29 \%$ with statin monotherapy and $40 \%$ with the combination therapy. In this small group of patients there was no significant increase in muscle, liver or renal toxicity or side effects. ${ }^{43} \mathrm{~A}$ number of other studies using combination treatment with statin and fenofibrate also revealed expected results on lipid parameters: all exhibited statistically significant decreases in TG, LDL, non-HDL, TC/HDL, in addition to hsCRP, and increases in HDL, with no increased organ toxicity. ${ }^{30,44,60}$ 
Farnier et al studied the combination of ezitimibe/ statin with fenofibrate in a multi-center double-blinded, placebo-controlled trial in a total of 641 subjects with combined dyslipidemia randomized to 4 different treatment arms. Combination therapy reduced LDL, TG and non-HDL levels by $46 \%, 50 \%$ and $51 \%$ in, respectively. ApoB was further reduced and HDL and apo A-1 levels increased with combination treatment. There was improvement in LDL pattern moving toward less dense larger particles (Pattern B to Pattern A). There was no significant increase in side effects or organ toxicity. ${ }^{47}$ A trial with fenofibrate $160 \mathrm{mg}$ /day combined with colesevelam $3.75 \mathrm{~g} /$ day (a bile acid sequestrant) produced greater reductions in LDL and apoB, without significant difference in HDL or TG, than fenofibrate alone. ${ }^{61}$ No significant safety issues were noted.

The clinical studies noted are consistent with significant $\mathrm{CV}$ benefits with fibrate use. However this frequently appears confusing to clinicians due to a variety of factors. The playing field that fibrates, or any additional lipid-modifying therapy, face includes massive, paradigm-changing, randomized trials using statin monotherapy commonly in broader and less complicated subject populations. Beneficial effects in fibrate trials have frequently been noted in subsets of patients, with fewer subjects therefore often looked on with unwarranted suspicion. Fibrates also have significant multiple lipid/ lipoprotein-modifying effects which may have substantially greater impact than realized to date. They have a myriad of other metabolic and vascular effects, as noted earlier, perhaps helping to regulate hundreds of genes, and probably play important roles that are not explained by their lipid-modifying effects, including improving insulin sensitivity and reducing vascular inflammatory processes.

\section{TriLipix $^{\circledR}$ : a new fibrate}

Previously available fenofibrates are pro-drugs which require de-esterification in the liver to fenofibric acid, the active drug, which is than released into the plasma bound to albumin and transported to tissues where $\operatorname{PPAR}(\alpha)$ is expressed (liver, vascular endothelium, adipocytes, muscle cells). The Food and Drug Administration (FDA) recently approved TriLipix ${ }^{\circledR}$ (Abbott Laboratories), a delayed-release choline salt of fenofibric acid which is not a pro-drug (Figure 7). The data presented are taken directly or consistent with the FDA-approved package insert. ${ }^{62}$ TriLipix requires no hepatic modification in order to activate $\operatorname{PPAR}(\alpha)$. It dissociates in the intestine releasing its choline group and active fenofibric acid. The formulation comes in 2 strengths, 45 and $135 \mathrm{mg}$. The capsules contain multiple mini-tablets containing hypromellose to extend release and are enterically coated to prevent dissociation in the acidic environment of the stomach. Fenofibric acid has poor solubility at gastric $\mathrm{pH}$ but excellent at intestinal $\mathrm{pH}$. Absolute bioavailability is in excess of $80 \%$. Peak plasma concentration occurs at 4 to 5 hours after oral administration. Phase I data reveal bioequivalence [peak concentration and area under the curve (AUC)] between $200 \mathrm{mg}$ of micronized fenofibrate and $135 \mathrm{mg}$ of TriLipix regardless of meals. The active drug, fenofibric acid, enters intestinal enterocytes in addition attaching to albumin and delivered to the liver, adipocytes and the vascular endothelium. The half-life of fenofibric acid is 20 hours allowing for once daily dosing.

The commonly used fibrates in the US are gemfibrozil and fenofibrate. Both gemfibrozil and fenofibrate undergo glucuronidation in the liver through different enzymatic pathways and are excreted mainly in the urine and to a minor degree in feces. Gemfibrozil has a significant competitive effect on statin, TZD and ezitimibe metabolism and is associated with a doubling of peak statin serum concentration and $\mathrm{AUC}$, increasing exposure and risk of muscle toxicity. Fenofibric acid does not compete with statin metabolism or increase statin levels and has no other known drug-drug interactions except modestly with warfarin. ${ }^{62-64}$ Like fenofibrate, TriLipix does not appear to increase risk of muscle, renal or hepatic toxicity. There are no differences in pharmacokinetics between males and females. Dose adjustment in those with mild to moderate renal impairment is recommended (45 $\mathrm{mg}$ dose). TriLipix has not been studied specifically in the elderly but the minimal amount of data available reveals similar pharmacokinetics, with slightly decreased $\mathrm{C}_{\max }$ (peak concentration) and AUC with slightly prolonged half-life compared with younger subjects. There are no recommended dosage adjustments in the geriatric population; however, since renal impairment is more likely in the elderly dose should be selected on the basis of renal function and subsequent monitoring should be performed. It has not been studied in the pediatric population.

TriLipix efficacy and safety when administered with statins was evaluated in three 12-week long, multi-center, placebo-controlled, double-blind Phase III studies. ${ }^{65-67}$ One 52-week, long-term, open-label extension study in 2698 patients with mixed dyslipidemia ${ }^{68}$ has also been reported. Each study compared the efficacy of (1) $135 \mathrm{mg} / \mathrm{dL}$ of TriLipix co-administered with either a low or intermediate-dose statin versus (2) TriLipix alone or (3) statin 


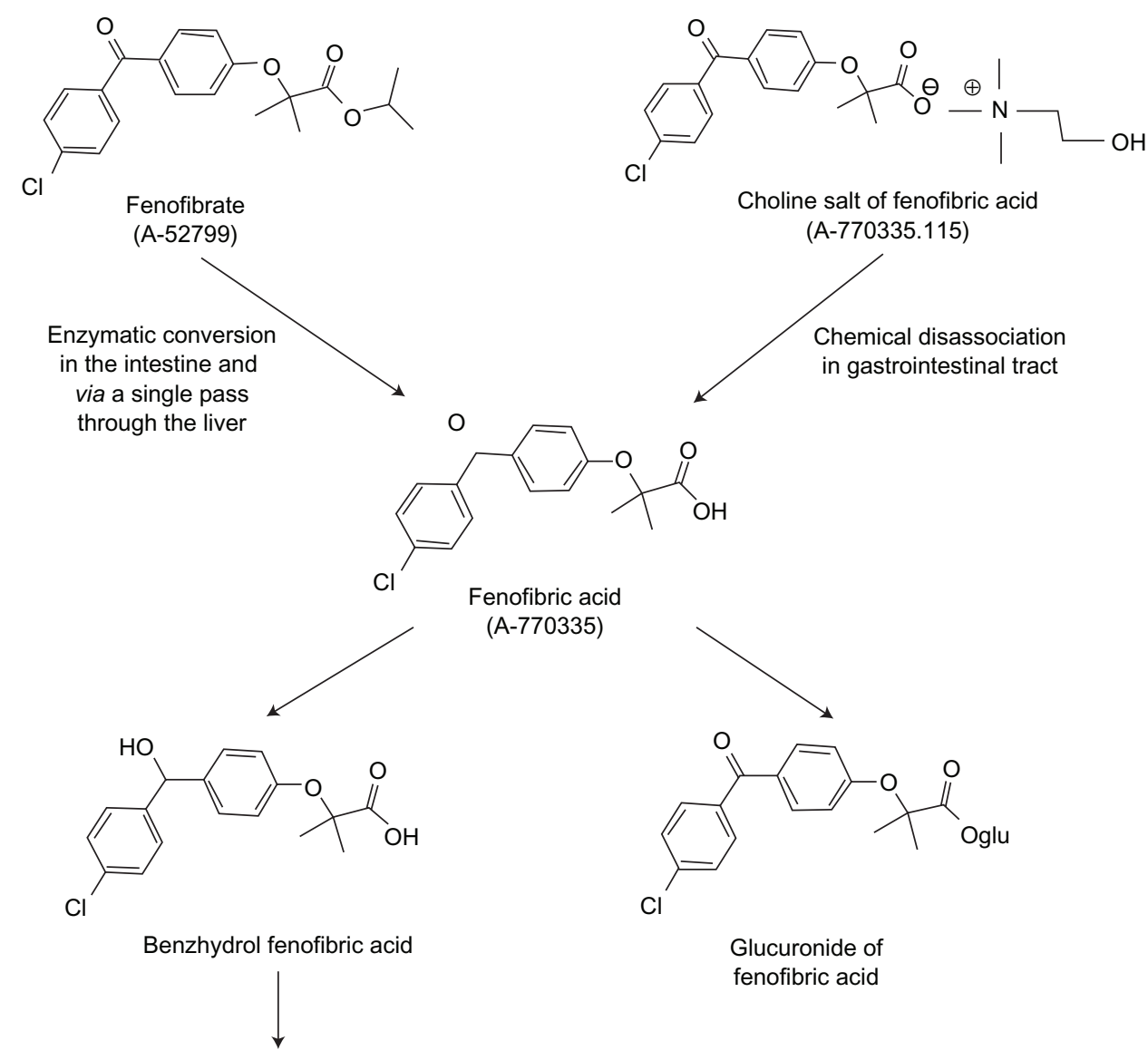

Glucuronide of benzhydrol fenofibric acid

Figure 7 Structure and metabolic pathway for fenofibrate and fenofibric acid (TriLipix) in humans.

monotherapy at the same dose on coronary heart disease lipid risk factors. A smaller group of patients received highdose statin monotherapy. The statin doses in each study were rosuvastatin 10 and $20 \mathrm{mg}$, simvastatin 20 and $40 \mathrm{mg}$, and atorvastatin 20 and $40 \mathrm{mg}$. The subjects were enrolled for 22 weeks which included a 6-week diet run-in/washout period, a 12-week treatment period, and a 30-day safety follow-up period. Patients who completed the 12 -week treatment period were eligible to participate in the 52-week long-term study. The mean age in all studies was 54.9 years and there was an equal distribution of men and women. The primary efficacy end-points for the studies were mean percent changes from baseline in HDL, TG, and LDL. Statistically significant positive differences were observed for all three efficacy comparisons at both doses of combination therapy in all studies versus either form of monotherapy. There were no differences in the safety profiles between the mono- and the combination therapies and no evidence of, or signals consistent with, hepatic or muscle toxicity were identified. Studies already noted using TriLipix in combination with statins improved overall atherogenic profiles in subjects with mixed or combined dyslipidemia as expected, without significantly increased side effect or safety concerns. There was no evidence of increased organ toxicity.

\section{Conclusion}

We appear to have reached maximum benefit (30\% to 35\% RRR for $\mathrm{CV}$ events) from the most aggressive statin monotherapy in the secondary risk population. Considering the tens of millions of potential patients at risk of $\mathrm{CV}$ disease and events, we should be curious as to how these results can be improved. There is substantial information on risk imparted by non-LDL factors including TG and HDL. Non-HDL has now been identified as a stronger risk predictor of $\mathrm{CV}$ events than LDL, and has an NCEP ATP defined goal. These factors can be addressed by a variety of pharmacologic approaches including combination therapy in addition to therapeutic lifestyle modification. The American Diabetes Association and the American College of Cardiology now recommend the consideration of combination lipid-modifying therapy, especially in those with elevated TG 
and non-HDL, in patients with CAD. ${ }^{69}$ TriLipix (fenofibric acid) recently received FDA approval for both mono- and combination therapy with low and medium dose statins. Trial data reveal excellent efficacy on lipid/lipoproteins as well as markers of inflammation, alone and in combination. Data do not reveal increased risk of muscle, renal or hepatic toxicity. This should encourage health care professionals, especially those concerned with prior lack of adequate safety data, to consider more aggressive lipid-modifying combination treatment with statin and fenofibric acid (TriLipix) in appropriate patients to further reduce residual risk for $\mathrm{CV}$ events.

\section{Disclosure}

The author has participated on Advisory Boards and speakers bureaus for a number of pharmaceutical companies including Abbott.

\section{References}

1. Castelli WP, Garrison RJ, Wilson PWF, et al. Incidence of coronary heart disease and lipoprotein cholesterol levels: The Framingham Study. JAMA. 1986;256:2835-2838.

2. Expert Panel on Detection, Evaluation, and Treatment of High Blood Cholesterol in Adults. Executive Summary of the Third Report of the National Cholesterol Education Program (Adult Treatment Panel III). JAMA. 2001;285:2486-2497.

3. Third Report of the National Cholesterol Education Program (NCEP) Expert Panel on Detection, Evaluation and Treatment of High Blood Cholesterol in Adults (Adult Treatment Panel III) final report. Circulation. 2002;106:3143-3421.

4. Cannon CP, Braunwald E, McCabe $\mathrm{CH}$, et al. Intensive versus moderate lipid lowering with statins after acute coronary syndromes. $N$ Engl J Med. 2004;350:1495-1504.

5. LaRosa JC, Grundy SM, Waters DD, et al. Intensive lipid lowering with atorvastatin in patients with stable coronary disease. $N$ Engl J Med. 2005;352:1425-1435.

6. Pedersen TR, Faergeman O, Kastelein JJ, et al. High dose atorvastatin vs usual-dose simvastatin for secondary prevention after myocardial infarction: the IDEAL study:a randomized controlled trial. JAMA. 2005;294:2437-2445.

7. Grundy SM, Cleeman JI, Merz CN, et al. Implications of recent clinical trials for the National Cholesterol Education Program Adult Treatment Panel III guidelines. Circulation. 2004;110:227-239.

8. Grundy SM. Statin trials and goals of cholesterol-lowering therapy. Circulation. 1998;97:1436-1439.

9. Downs JR, Clearfield M, Weis S, et al. Primary prevention of acute coronary events with lovastatin in men and women with average cholesterol levels: Results of AFCAPS/TexCAPS. JAMA. 1998;279:1615-1622.

10. Shepherd J, Cobbe SM, Ford I, et al. Prevention of coronary heart disease with pravastatin in men with hypercholesterolemia. $N$ Engl J Med. 1995;333:1301-1307.

11. Hayward RA, Hofer TP, Vijan S. Narrative review: lack of evidence for recommended low-density lipoprotein treatment targets: a solvable problem. Ann Intern Med. 2006;145:520-530.

12. Tabas I, Williams KJ, Boren J. Subendothelial lipoprotein retention as the initiating process in atherosclerosis: update and therapeutic implications. Circulation. 2007;116:1832-1844.

13. Koschinsky ML, Marcovina SM. In: Ballantyne, CM, editor. Clinical Lipidology: A Companion to Braunwald's Heart Disease. Philadelphia: Saunders Elsevier; 2009:130-143.
14. Grundy SM. Atherogenic dyslipidemia associated with metabolic syndrome and insulin resistance. Clin Cornerstone. 2006;8 Suppl 1:S21-S27.

15. Castelli WP. Cholesterol and lipids in the risk of coronary heart disease-the Framingham Heart Study. Can J Cardiol. 1988;4 Suppl A:5A-10A.

16. Castelli WP. Epidemiology of triglycerides: a view from Framingham. Am J Cardiol. 1992;70:3H-9H.

17. Gordon DJ, Probstfield JL, Garrison RJ, et al. High density lipoprotein cholesterol and cardiovascular disease. Four prospective American studies. Circulation. 1989;79:8-15.

18. Hokanson JE and Austin MA. Triglyceride is a risk factor for cardiovascular disease independent of high-density lipoprotein: a meta-analysis of population-based prospective studies. J Cardiovasc Risk. 1996;3:213-219.

19. Yarnell JWG, Patterson CC, Sweetnam PM, et al. Do total and high density lipoprotein cholesterol and triglycerides act independently in the prediction of ischemic heart disease. Arterioscler Thromb Vasc Biol. 2001;21:1340-1345.

20. Jeppesen J, Hein HO, Suadicani P, Gyntelberg F. Clinical investigation and reports: Triglyceride concentration and ischemia heart disease. An eight-year follow-up in the Copenhagen Male Study. Circulation. 1998;97:1029-1036.

21. Assmann G, Schulte H and Eckardstein A. Hypertriglyceridemia and elevated lipoprotein(a) are are risk factors for major coronary events in middle-aged men. Am J Cardiol. 1996;77:1179-1184.

22. Genest JJ, Martin-Munley SS, McNamara JR, et al. Familial lipoprotein disorders in patients with premature coronary artery disease. Circulation. 1992;85:2025-2033.

23. Genest JJ, McNamara JR, Salem DN, Shaefer, EJ. Prevalence of risk factors in men with premature coronary artery disease. Am J Cardiol. 1991;67:1185-1189.

24. Gordon T, Castelli WP, Hjortland MC, et al. High density lipoprotein as a protective factor against coronary heart disease. The Framingham Study. Am J Med. 1977;62:707-714.

25. Liu J, Sempos CT, Donahue RP, et al. Non-high density lipoprotein and very low density lipoprotein cholesterol and their risk predictive values in coronary heart disease. Am J Cardiol. 2006;98: 1363-1368.

26. Frick MH, Elo MO, Happa K, et al. Helsinki Heart Study: primary-prevention trial with gemfibrozil in middle-aged men with dyslipidemia. Safety of treatment, changes in risk factors, and incidence of coronary heart disease. N Engl J Med. 1987;317:1237-1245.

27. Haim M, Benderly M, Brunner D, et al. Elevated serum triglyceride levels in patients with coronary heart disease: The bezafibrate Infarction Prevention (BIP) Registry. Circulation. 1999;100:475-482.

28. Carlson LA, Rosenhaner G. Reduction of mortality in the in the Stockholm Ischaemic Heart Disease Secondary Prevention Study by combined treatment with clofibrate and nicotinic acid. Acta Med Scand. 1988;223:405-418.

29. Rubins HB, Robins SJ, Collins D, et al. Gemfibrozil for the secondary prevention of coronary heart disease in men with low high-density cholesterol. N Engl J Med. 1999;341:410-418.

30. Keech A, Simes RJ, Barter P, et al. The Field Study Investigators. Effects of long-term fenofibrate therapy on cardiovascular events in 9795 people with type 2 diabetes mellitus (the FIELD study): randomised control trial. Lancet. 2005;366:1849-1861.

31. The "Effects of Combination Lipid Therapy in Type 2 Diabetes Mellitus" an NIH study with support from multiple industry partners was just published (Effects of Combination Therapy in Type 2 Diabetes Mellitus. The ACCORD Study Group. NEngl J of Med; March 14, 2010, and updated on March 18, 2010, at NEJM.org (10.1056/NEJMoa1001282).

32. Superko HR, King S III. Is lowering low-density lipoprotein an effective strategy to reduce cardiac risk. Circulation. 2008;117:560-568.

33. Staelas B, Dallongville J, Auwerx J, et al. Mechanism of action of fibrates on lipid and lipoprotein metabolism. Circulation. 1998;98:2088-2093.

34. Berger J, Moeller D. The mechanisms of action of PPARs. Annu Rev Med. 2002;53:409-435. 
35. Staels B, Auwerx J. Regulation of apoA-1 gene expression by fibrates. Atherosclerosis. 1998; Suppl 137:S19-S23.

36. Chinetti G, Lastavel S, Bocker V, et al. PPAR-alpha and PPAR gamma activators induce cholesterol removal from human macrophages from cells through stimulation of the ABCA1 pathway. Nat Med. 2001;7:53-58

37. Marx N, Sukhova GK, Collins T, et al. PPAR alpha activators inhibit cytokine-induced vascular cell adhesion molecule-1 expression in human endothelial cells. Circulation. 1999;99:3125-3131.

38. Marx N, Mackman N, Schonbeck U, et al. PPAR alpha agonists inhibit tissue factor expression in human monocytes and macrophages. Circulation. 2001;103:213-219.

39. Jones, PH. Fibrates. In: Ballantyne, CM, editor. Clinical Lipidology: A Companion to Braunwald's Heart Disease. Philadelphia: Saunders Elsevier; 2009:315-325.

40. Muhlestein JB, May HT, Jensen JR, et al. The reduction of inflammatory biomarkers by statin, fibrate and combination therapy among diabetic patients with mixed dyslipidemia. The DIACOR (Diabetes and Combined Lipid Therapy Regimen) study. J Am Coll Cardiol. 2006;48:396-401.

41. Koh KK, Han SH, Quon MJ, et al. Beneficial effects of fenofibrate to improve endothelial dysfunction and raise adiponectin levels in patients with primary hypertriglyceridemia. Diabetes Care. 2005;28:1419-1424.

42. Wysocki J, Belowski D, Kalina M, et al. Effects of micronized fenofibrate on insulin resistance in patients with metabolic syndrome. Int J Clin Pharmacol Ther. 2004;42:212-217.

43. Ellen RLB, McPherson R. Long-term efficacy and safety of fenofibrate and a statin in the treatment of combination hyperlipidemia. Am J Cardiol. 1998;81(4A):60B-65B.

44. Grundy SM, Vega GL, Yuan Z, et al. Effectiveness and tolerability of simvastatin plus fenofibrate for combined hyperlipidemia (The Safari Trial). Am J Cardiol. 2005;95:462-468.

45. Davidson MH, Armani A, McKenney JM, et al. Safety considerations with fibrate therapy. Am J Cardiol. 2007; Suppl 99:3C-18C.

46. Farnier M, Freeman MW, Macdonell G, et al. Efficacy and safety of the coadministration of ezetimibe with fenofibrate in patients with mixed hyperlipidaemia. Eur Heart J. 2005;26:897-905.

47. Farnier M, Roth E, Gil-Extremera B, et al. Efficacy and safety of the coadministration ezetimibe/simvastatin with fenofibrate in patients with mixed hyperlipidemia. Am Heart J. 2007;153:335.e1-335.e8.

48. Rizos E, Biaraktari E, Ganotakis E, et al. Effect of ciprofibrate on lipoproteins, fibrinogen, renal function, and hepatic enzymes. J Cardiovasc Pharmacol Ther. 2002;7:219-226.

49. Keech A, Simes RJ, Barter P, et al. Effects of long-term fenofibrate therapy on cardiovascular events 9795 people with type II diabetes mellitus (the FIELD study): Randomized control trial. Lancet. 2005;366:1849-1861.

50. Hottelart C, El Esper N, Rose F, et al. Fenofibrate increased creatinemia by increasing metabolic production of creatinine. Nephron. 2002;92:536.

51. Tsimihodimos V, Miltiadous G, Bairaktari E and Elisaf M. Possible mechanisms of the fibrate-induced increase in serum creatinine. Clin Nephrol. 2002;57:407-408.

52. Tonelli M, Collins D, Robins S, et al. Effect of gemfibrozil on change in renal function in men with moderate chronic renal insufficiency and coronary disease. Am J Kidney Dis. 2004;44:832-839.
53. Ansquen JC, Foucher C, Rattier S, et al. Fenofibrate reduces progression to microalbuminuria over 3 years in a placebo-controlled study in Type 2 diabetes: Results from the Diabetes Atherosclerosis Intervention Study (DAIS). Am J Kidney Dis. 2005;45:485-493.

54. Mekkies M, Stein E, Khoury P, et al. Effects of fenofibrate on lipids, lipoproteins and apolipoproteins in 33 subjects with primary hypercholesterolaemia. Atherosclerosis. 1987;63:57-64.

55. Bradford R, Goldberg A, Schoenfeld G, et al. Double-blind comparison of bezafibrate versus placebo in male volunteers with hyperlipoproteinemia. Atherosclerosis. 1992;92: $31-40$.

56. Gotto AM, Breen WJ, Cordner CN, et al. Once-daily, extended-release gemfibrozil in patients with dyslipidemia. The Lopid SR Work Group I. Am J Cardiol. 1993;71:1057-1063.

57. Zimetbaum P, Frishman WH, Kahn S. Effects of gemfibrozil and other fibric acid derivatives on blood lipids and lipoproteins. J Clin Pharmacol. 1991;31:25-37.

58. Staels B, Dallongeville J, Auwerx J, et al. Mechanism of action of fibrates on lipid and lipoprotein metabolism. Circulation. 1998;98:2088-2093.

59. Lahdenpera S, Tilly-Kiesi M, Vuorinen-Markkola H, et al. Effects of gemfibrozil on low-density lipoprotein particle size, density, distribution and composition in patients with type II diabetes. Diabetes Care. 1993; 16:584-592.

60. Koh KK, Quon MJ, Han SH, et al. Additive beneficial effects of fenofibrate combined with atorvastatin in the treatment of combined hyperlipidemia. J Am Coll Cardiol. 2005;45:1649-1953.

61. McKenney J, Jones M, Abby S. Safety and efficacy of colesevelam hydrochloride in combination with fenofibrate for the treatment of mixed hyperlipidemia. Curr Med Res Opin. 2005;21:1403-1412.

62. Trilipix [package insert]. North Chicago (IL): Abbott Laboratories; 2008.

63. Jones PH, Davidson MH. Reporting rate of rhabdomyolysis with fenofibrate + fibrates versus gemfibrozil + any statin. Am J Cardiol. 2005;95:120-122.

64. Jones PH, Bays HE, Davidson MH, et al. Evaluation of a new formulation of fenofibric acid, ABT-335, co-administered with statins. Clin Drug Investig. 2008;28:625-634.

65. Jones PH, Davidson MH, Kashyap ML, et al. Efficacy and safety of ABT-335 (fenofibric acid) in combination with rosuvastatin in patients with mixed dyslipidemia: A phase 3 study. Atherosclerosis. 2009;204:208-215.

66. Mohiuddin SM, Pepine CJ, Kelly MT, et al. Efficacy and safety of ABT-335 fenofibric acid in combination with simvastatin in patients with mixed dyslipidemia: A phase 3, randomized, control study. Am Heart J. 2009;157:195-203.

67. Goldberg AC, Bays HE, Ballantyne CM, et al. Efficacy and safety of ABT-335 (fenofibric acid) in combination with atorvastatin in patients with mixed dyslipidemia. Am J Cardiol. 2009;103:515-522.

68. Bays HE, Jones PH, Mohiuddin SM, et al. Long-term safety and efficacy of fenofibric acid in combination with statin therapy for the treatment of patients with mixed dyslipidemia. J Clin Lipidol. 2008;2:426-435.

69. Brunzell JD, Davidson M, Furberg CD, et al. Lipoprotein management in patients with cardiometabolic risk: consensus statement from the American Diabetes Association and the American College of Cardiology Foundation. Diabetes Care. 2008;31:811-822.
Vascular Health and Risk Management

\section{Publish your work in this journal}

Vascular Health and Risk Management is an international, peerreviewed journal of therapeutics and risk management, focusing on concise rapid reporting of clinical studies on the processes involved in the maintenance of vascular health; the monitoring, prevention and treatment of vascular disease and its sequelae; and the involvement of

\section{Dovepress}

metabolic disorders, particularly diabetes. This journal is indexed on PubMed Central and MedLine. The manuscript management system is completely online and includes a very quick and fair peer-review system, which is all easy to use. Visit http://www.dovepress.com/ testimonials.php to read real quotes from published authors. 